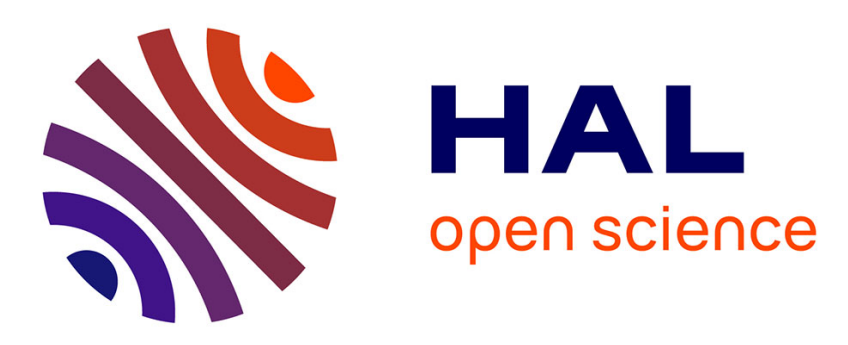

\title{
Inversion of higher order isotropic tensors with minor symmetries and solution of higher order heterogeneity problems
}

\author{
Vincent Monchiet, Guy Bonnet
}

\section{- To cite this version:}

Vincent Monchiet, Guy Bonnet. Inversion of higher order isotropic tensors with minor symmetries and solution of higher order heterogeneity problems. Proceedings of the Royal Society of London A, 2011, 467 (2126), pp.314-332. hal-00687817

\section{HAL Id: hal-00687817 https://hal.science/hal-00687817}

Submitted on 15 Apr 2012

HAL is a multi-disciplinary open access archive for the deposit and dissemination of scientific research documents, whether they are published or not. The documents may come from teaching and research institutions in France or abroad, or from public or private research centers.
L'archive ouverte pluridisciplinaire HAL, est destinée au dépôt et à la diffusion de documents scientifiques de niveau recherche, publiés ou non, émanant des établissements d'enseignement et de recherche français ou étrangers, des laboratoires publics ou privés. 


\title{
Inversion of higher order isotropic tensors with minor symmetries and solution of higher order heterogeneity problems
}

\author{
By Vincent Monchiet, Guy Bonnet \\ Université Paris-Est, Laboratoire Modélisation et Simulation Multi Echelle, \\ LMSME UMR8208 CNRS, 5 boulevard Descartes, 77454 Marne la Vallée Cedex,
}

France

In this paper, the derivation of irreducible bases for a class of isotropic $(2 n)^{t h}$ order tensors having particular "minor symmetries" is presented. The methodology used for obtaining these bases consists in extending the concept of deviatoric and spherical parts, commonly used for $2^{n d}$-order tensors, to the case of a $n^{t h}$-order tensor. It is shown that those bases are useful for effecting the classical tensorial operations and specially the inversion of a $2 n^{\text {th }}$-order tensor. Finally, the formalism introduced in this study is applied for obtaining the closed form expression of the strain field within a spherical inclusion embedded in an infinite elastic matrix and subjected to linear or quadratic polynomial remote strain fields.

Keywords: Isotropic tensors, irreducible basis, inclusion problem

\section{Introduction}

Some specific problem in mechanics take the form of linear equations between two tensors having an order higher than $n=2$. For instance, the theories of generalized continuum (Toupin 1962, Mindlin 1964, Mindlin \& Eshel 68, Suiker \& Chang 2000) introduce higher order gradients of the displacement for the description of the continuum. The generalized gradient elastic constitutive equation introduces then tensors of order $6,8 \ldots$ More recently such considerations were extended to nonlinear elasticity (Dell'Isola et al. 2009). From another point of view, the problem of an elastic inclusion embedded in an infinite elastic medium and subjected to a polynomial remote strain field has been studied by Asaro \& Barnett 1975, Mura 1987. It has been shown that the complete solution requires to solve a linear system involving the inversion of tensors of order $6,8, \ldots$ However, there are no explicit known closed forms for the inverses of such higher order tensors.

To summarize, in all the problems quoted above, two $n^{\text {th }}$-order tensors $\boldsymbol{a}$ and $\boldsymbol{b}$ are related through a linear relation which takes the form:

$$
\boldsymbol{b}=\mathbb{A} \odot_{n} \boldsymbol{a}
$$

Where $\mathbb{A}$ is a tensor of order $2 n$. We assume that the components of $\boldsymbol{a}$ and $\boldsymbol{b}$ are symmetric according to their two first indices $a_{i j p . . q}=a_{j i p . . q}, b_{i j p . . q}=b_{j i p . . q}$ and are invariant by any permutation of their $n-2$ last indices $p . . q$. For instance, in 
the case $n=3,4,5$ one has:

$$
\begin{array}{ll}
n=3: & a_{i j p}=a_{j i p} \\
n=4: & a_{i j p q}=a_{j i p q} \\
& a_{i j p q}=a_{i j q p} \\
n=5: & a_{i j p q r}=a_{j i p q r} \\
& a_{i j p q r}=a_{i j p r q}=a_{i j q p r}=a_{i j q r p}=a_{i j r p q}=q_{i j r q p}
\end{array}
$$

etc.

In relation (1.1), $\odot_{n}$ denotes the $n^{t h}$ contraction between $\mathbb{A}$ and $\boldsymbol{a}$ such that $b_{i j p . . q}=$ $A_{i j p . . q k l r . . s} a_{k l r . . s}$. Due to the symmetries of $\boldsymbol{a}$ and $\boldsymbol{b}, A_{i j p . . q k l r . . s}$ is invariant by any permutation of indices $(i, j),(k, l),(p, . ., q)$ and $(r, . ., s)$. Through this paper, these symmetries are called "minor symmetries". Note that $\mathbb{A}$ does not necessarily possess the "major symmetry", namely $A_{i j p . . q k l r . . s} \neq A_{k l r . . s i j p . . q}$.

In the present study, we assume that tensor $\mathbb{A}$ is isotropic and we denote by $E_{2 n}$ the space of isotropic $2 n^{t h}$-order tensors having the minor symmetries. We propose to build the inverse of $\mathbb{A}$, namely the tensor $\mathbb{B}$ such that:

$$
\boldsymbol{a}=\mathbb{B} \odot_{n} \boldsymbol{b}
$$

In the case $n=2, \mathbb{A}$ is an isotropic tensor of $4^{\text {th }}$ order. Due to symmetries mentioned above, $\mathbb{A}$ depends on two independent coefficients $a_{1}$ and $a_{2}$ and can be expressed as $\mathbb{A}=a_{1} \mathbb{J}+a_{2} \mathbb{K}$. Tensors $\mathbb{J}$ and $\mathbb{K}$ are defined by $J_{i j k l}=\delta_{i j} \delta_{k l} / 3, K_{i j k l}=I_{i j k l}-J_{i j k l}$ and $I_{i j k l}=\left(\delta_{i k} \delta_{j l}+\delta_{i l} \delta_{j k}\right) / 2$ where $\delta_{i j}$ is the Kronecker symbol. $\mathbb{J}$ and $\mathbb{K}$ are two projectors which define an irreducible basis for isotropic tensors having the minor symmetries. The use of these two tensors produces easily the inversion of $\mathbb{A}$ since $\mathbb{B}=1 / a_{1} \mathbb{J}+1 / a_{2} \mathbb{K}$.

We aim at extending this basis in the case of higher order isotropic tensors. The paper is organized as follows: in section 2 , we first state about the case $n=3$. An irreducible basis, constituted of six independent tensors, is obtained and appears to be convenient for effecting the classical tensorial operations and specially the inversion. The methodology applied for obtaining this basis is clearly depicted in this section. It consists in generalizing the concept of deviatoric and spherical part, commonly used for second order tensors, to the case of a tensor of order 3. This approach is afterwards applied to the case of a $8^{t h}$-order tensor. Its generalization to the case of a tensor of order up to $2 n=8$ is addressed in section 4 . In fine, the application of the methodology to the inclusion problem is performed.

\section{A basis for $6^{\text {th }}$-order isotropic tensors}

In this section, we first consider the case $n=3$ in (1.1), $\boldsymbol{a}$ and $\boldsymbol{b}$ are then two third-order tensors while $\mathbb{A}$ is a sixth order isotropic tensor. $\mathbb{A}$ has the symmetries: $A_{i j k p q r}=A_{j i k p q r}, A_{i j k p q r}=A_{i j k q p r}$. Tensor $\mathbb{A}$ is invariant under the orthogonal group $\mathrm{O}_{3}$, consequently:

$$
A_{i . . j}=Q_{i p . .} Q_{j q} A_{p . . q}
$$


$Q_{i p} . . Q_{j q}$ are the orthogonal matrices of the group $O_{3}$, which satisfy $Q_{i k} Q_{j k}=\delta_{i j}$ and $\operatorname{det}(Q)= \pm 1$. Every isotropic tensor of order $2 n$ ( $n$ being an integer) can be expressed in terms of the Kronecker symbol. Particularly, a sixth order isotropic tensor can be read as a linear combination of:

$$
\begin{aligned}
& \delta_{i j} \delta_{k r} \delta_{p q}, \delta_{i j} \delta_{k p} \delta_{q r}, \delta_{i j} \delta_{k q} \delta_{p r}, \delta_{i k} \delta_{j r} \delta_{p q}, \delta_{i k} \delta_{j p} \delta_{q r} \\
& \delta_{i k} \delta_{j q} \delta_{p r}, \delta_{i p} \delta_{j k} \delta_{q r}, \delta_{i p} \delta_{j r} \delta_{k q}, \delta_{i p} \delta_{j q} \delta_{k r}, \delta_{i q} \delta_{j k} \delta_{p r} \\
& \delta_{i q} \delta_{j r} \delta_{k p}, \delta_{i q} \delta_{j p} \delta_{k r}, \delta_{i r} \delta_{j k} \delta_{p q}, \delta_{i r} \delta_{j p} \delta_{k q}, \delta_{i r} \delta_{j q} \delta_{k p}
\end{aligned}
$$

For a tensor having the minor symmetries, only six tensors are needed. They are denoted: $\mathbb{T}_{1}, \ldots, \mathbb{T}_{6}$ and their components are:

$$
\begin{aligned}
& \left(\mathbb{T}_{1}\right)_{i j k p q r}=\delta_{i j} \delta_{p q} \delta_{k r}, \quad\left(\mathbb{T}_{2}\right)_{i j k p q r}=I_{i j p q} \delta_{k r} \\
& \left(\mathbb{T}_{3}\right)_{i j k p q r}=I_{i j k r} \delta_{p q}, \quad\left(\mathbb{T}_{4}\right)_{i j k p q r}=I_{p q k r} \delta_{i j} \\
& \left(\mathbb{T}_{5}\right)_{i j k p q r}=\frac{1}{2}\left(I_{i j p r} \delta_{k q}+I_{i j q r} \delta_{k p}\right), \quad\left(\mathbb{T}_{6}\right)_{i j k p q r}=\frac{1}{2}\left(I_{p q i r} \delta_{j k}+I_{p q j r} \delta_{i k}\right)
\end{aligned}
$$

where it is recalled that $I_{i j k l}=\left(\delta_{i k} \delta_{j l}+\delta_{i l} \delta_{j k}\right) / 2$.

The triple contraction between two tensors taken from $\left(\mathbb{T}_{1}, . ., \mathbb{T}_{6}\right)$ are given in table 1. $\left(\mathbb{T}_{1}, \ldots, \mathbb{T}_{6}\right)$ constitute a basis for all tensors $\mathbb{A} \in E_{6}$. However, this basis is not convenient for the inversion of $6^{\text {th }}$-order tensors since it leads to a complex linear system of dimension 6 .

\begin{tabular}{|c|c|c|c|c|c|c|}
\hline$\odot_{3}$ & $\mathbb{T}_{1}$ & $\mathbb{T}_{2}$ & $\mathbb{T}_{3}$ & $\mathbb{T}_{4}$ & $\mathbb{T}_{5}$ & $\mathbb{T}_{6}$ \\
\hline $\mathbb{T}_{1}$ & $3 \mathbb{T}_{1}$ & $\mathbb{T}_{1}$ & $\mathbb{T}_{1}$ & $3 \mathbb{T}_{4}$ & $\mathbb{T}_{4}$ & $\mathbb{T}_{4}$ \\
\hline $\mathbb{T}_{2}$ & $\mathbb{T}_{1}$ & $\mathbb{T}_{2}$ & $\mathbb{T}_{3}$ & $\mathbb{T}_{4}$ & $\mathbb{T}_{5}$ & $\mathbb{T}_{6}$ \\
\hline $\mathbb{T}_{3}$ & $3 \mathbb{T}_{3}$ & $\mathbb{T}_{3}$ & $\mathbb{T}_{3}$ & $3 \mathbb{T}_{6}$ & $\mathbb{T}_{6}$ & $\mathbb{T}_{6}$ \\
\hline $\mathbb{T}_{4}$ & $\mathbb{T}_{1}$ & $\mathbb{T}_{4}$ & $2 \mathbb{T}_{1}$ & $\mathbb{T}_{4}$ & $\frac{1}{2}\left(\mathbb{T}_{1}+\mathbb{T}_{4}\right)$ & $2 \mathbb{T}_{4}$ \\
\hline $\mathbb{T}_{5}$ & $\mathbb{T}_{3}$ & $\mathbb{T}_{5}$ & $\frac{1}{2}\left(\mathbb{T}_{1}+\mathbb{T}_{3}\right)$ & $\mathbb{T}_{6}$ & $\frac{1}{2}\left(\mathbb{T}_{2}+\mathbb{T}_{5}\right)$ & $\frac{1}{2}\left(\mathbb{T}_{4}+\mathbb{T}_{6}\right)$ \\
\hline $\mathbb{T}_{6}$ & $\mathbb{T}_{3}$ & $\mathbb{T}_{6}$ & $2 \mathbb{T}_{3}$ & $\mathbb{T}_{6}$ & $\frac{1}{2}\left(\mathbb{T}_{3}+\mathbb{T}_{6}\right)$ & $2 \mathbb{T}_{6}$ \\
\hline
\end{tabular}

Table 1: The triple contraction between $\mathbb{T}_{n}$ and $\mathbb{T}_{m}$

In order to provide a simplified basis, we first introduce the spherical part, $S(\boldsymbol{a})$, and the deviatoric part, $D(\boldsymbol{a})$, of a $3^{\text {rd }}$-order tensor $\boldsymbol{a}$ as follows:

$$
\begin{aligned}
D(\boldsymbol{a}) & =\boldsymbol{a}-S(\boldsymbol{a}) \\
S(\boldsymbol{a}) & =\frac{1}{5}\left(2 a_{p p k}-a_{k p p}\right) \delta_{i j} \\
& +\frac{1}{10}\left(3 a_{i p p}-a_{p p i}\right) \delta_{j k}+\frac{1}{10}\left(3 a_{j p p}-a_{p p j}\right) \delta_{i k}
\end{aligned}
$$

$S(\boldsymbol{a})$ has the properties: $(S(\boldsymbol{a}))_{i p p}=a_{i p p}$ and $(S(\boldsymbol{a}))_{p p i}=a_{p p i}$. Consequently, all contractions of indices of $(D(\boldsymbol{a}))$ are null : $(D(\boldsymbol{a}))_{i p p}=(D(\boldsymbol{a}))_{p p i}=0$. It is 
therefore natural to consider $D(\boldsymbol{a})$ as the generalization to third-order tensors of the deviatoric part, which is well known for second order tensors. This decomposition suggests introducing the $6^{\text {th }}$-order tensors $\mathbb{J}, \mathbb{K}$ and $\mathbb{I}$ given by:

$$
\mathbb{J}=\frac{1}{5}\left(2 \mathbb{T}_{1}-\mathbb{T}_{3}-\mathbb{T}_{4}+3 \mathbb{T}_{6}\right), \quad \mathbb{K}=\mathbb{I}-\mathbb{J}, \quad \mathbb{I}=\mathbb{T}_{2}
$$

These tensors are such that $D(\boldsymbol{a})=\mathbb{K} \odot_{3} \boldsymbol{a}, S(\boldsymbol{a})=\mathbb{J} \odot_{3} \boldsymbol{a}, \boldsymbol{a}=\mathbb{I} \odot_{3} \boldsymbol{a}$. Here, $\mathbb{I}$ is the identity for the triple contraction $\odot_{3}$ and $\mathbb{J}$ and $\mathbb{K}$ produce the deviatoric and spherical parts of $\boldsymbol{a}$. From another point of view, every $6^{\text {th }}$ order isotropic tensor $\mathbb{A}$, having the minor symmetries is defined by 6 independent coefficient. Due to the previous relations it is natural to introduce the decomposition: $\mathbb{A}=\mathbb{A}_{J}+\mathbb{A}_{K}$ where $\mathbb{A}_{J}=\mathbb{J} \odot_{3} \mathbb{A}$ and $\mathbb{A}_{K}=\mathbb{K} \odot_{3} \mathbb{A}$. It is easy to show that tensor $\mathbb{A}_{J}$ is defined by 4 independent coefficients. It suggests therefore that there exist four tensors $\mathbb{J}_{n}$ with $n=1,2,3,4$ such that: $\mathbb{A}_{J}=a_{1} \mathbb{J}_{1}+a_{2} \mathbb{J}_{2}+a_{3} \mathbb{J}_{3}+a_{4} \mathbb{J}_{4}$ and $\mathbb{K} \odot_{3} \mathbb{J}_{n}=\mathbb{J}_{n} \odot_{3} \mathbb{K}=0$ whatever the value of $n$. In other words, we search $\mathbb{J}_{n}$ defined by $\mathbb{J}_{n}=\sum_{n} a_{n} \mathbb{T}_{n}$ such that: $\mathbb{J}_{n} \odot_{3} \mathbb{K}=\mathbb{K} \odot_{3} \mathbb{J}_{n}=0$ and $\mathbb{J}_{n} \odot_{3} \mathbb{J}=\mathbb{J} \odot_{3} \mathbb{J}_{n}=\mathbb{J}_{n}$. For its part, tensor $\mathbb{A}_{K}$ is defined by 2 independent coefficients. It suggests that there exist two tensors $\mathbb{K}_{1}$ and $\mathbb{K}_{2}$ such that $\mathbb{A}_{K}=a_{1} \mathbb{K}_{1}+a_{2} \mathbb{K}_{2}$ and $\mathbb{J} \odot_{3} \mathbb{K}_{n}=\mathbb{K}_{n} \odot_{3} \mathbb{J}=0$, $\mathbb{K} \odot_{3} \mathbb{K}_{n}=\mathbb{K}_{n} \odot_{3} \mathbb{K}=\mathbb{K}_{n}$ whatever $n=1,2$.

The following expressions were found for $\mathbb{J}_{n}$ and $\mathbb{K}_{n}$ :

$$
\begin{aligned}
& \mathbb{J}_{1}=\frac{1}{5}\left(2 \mathbb{T}_{1}-\mathbb{T}_{4}\right), \quad \mathbb{J}_{2}=\frac{1}{5}\left(3 \mathbb{T}_{4}-\mathbb{T}_{1}\right) \\
& \mathbb{J}_{3}=\frac{1}{5}\left(2 \mathbb{T}_{3}-\mathbb{T}_{6}\right), \quad \mathbb{J}_{4}=\frac{1}{5}\left(3 \mathbb{T}_{6}-\mathbb{T}_{3}\right) \\
& \mathbb{K}_{1}=\frac{1}{3}\left(\mathbb{T}_{2}+2 \mathbb{T}_{5}\right)-\frac{1}{3}\left(\mathbb{J}_{1}+\mathbb{J}_{2}\right)-\frac{2}{3}\left(\mathbb{J}_{3}+\mathbb{J}_{4}\right) \\
& \mathbb{K}_{2}=\frac{2}{3}\left(\mathbb{T}_{2}-\mathbb{T}_{5}\right)-\frac{1}{3}\left(2 \mathbb{J}_{1}+\mathbb{J}_{4}\right)+\frac{1}{3}\left(\mathbb{J}_{2}+2 \mathbb{J}_{3}\right)
\end{aligned}
$$

Note that $\mathbb{J}=\mathbb{J}_{1}+\mathbb{J}_{4}$ and $\mathbb{K}=\mathbb{K}_{1}+\mathbb{K}_{2}$.

In addition, the triple contraction between the different tensors $\mathbb{J}_{n}$ and $\mathbb{K}_{n}$ are given in table 2 :

\begin{tabular}{|c|c|c|c|c|c|c|}
\hline$\odot_{3}$ & $\mathbb{K}_{1}$ & $\mathbb{K}_{2}$ & $\mathbb{J}_{1}$ & $\mathbb{J}_{2}$ & $\mathbb{J}_{3}$ & $\mathbb{J}_{4}$ \\
\hline $\mathbb{K}_{1}$ & $\mathbb{K}_{1}$ & 0 & 0 & 0 & 0 & 0 \\
\hline $\mathbb{K}_{2}$ & 0 & $\mathbb{K}_{2}$ & 0 & 0 & 0 & 0 \\
\hline $\mathbb{J}_{1}$ & 0 & 0 & $\mathbb{J}_{1}$ & $\mathbb{J}_{2}$ & 0 & 0 \\
\hline $\mathbb{J}_{2}$ & 0 & 0 & 0 & 0 & $\mathbb{J}_{1}$ & $\mathbb{J}_{2}$ \\
\hline $\mathbb{J}_{3}$ & 0 & 0 & $\mathbb{J}_{3}$ & $\mathbb{J}_{4}$ & 0 & 0 \\
\hline $\mathbb{J}_{4}$ & 0 & 0 & 0 & 0 & $\mathbb{J}_{3}$ & $\mathbb{J}_{4}$ \\
\hline
\end{tabular}

Table 2: Triple contraction between $\mathbb{J}_{n}$ and $\mathbb{K}_{n}$

These results call the following remarks:

- It can be observed that, $\left(E_{6}, \odot_{3}, \mathbb{I}\right)$ define a monoid (an algebraic structure with a single associative binary operation and an identity element). $\mathbb{I}$ is the 
identity for the composition $\odot_{3}$ and is defined by (2.5). The six elements $\left(\mathbb{K}_{n}, \mathbb{J}_{m}\right)$ for $n=1,2$ and $m=1,2,3,4$ constitute an irreducible basis for $\left(E_{6}, \odot_{3}, \mathbb{I}\right)$. Every tensor $\mathbb{A} \in E_{6}$ can be read:

$$
\mathbb{A}=a_{1} \mathbb{K}_{1}+a_{2} \mathbb{K}_{2}+a_{3} \mathbb{J}_{1}+a_{4} \mathbb{J}_{2}+a_{5} \mathbb{J}_{3}+a_{6} \mathbb{J}_{4}
$$

- Introducing $K_{6}$, the space of isotropic $6^{\text {th }}$-order tensors given by $\mathbb{A}=a_{1} \mathbb{K}_{1}+$ $a_{2} \mathbb{K}_{2}$, it can be also shown from table 2 , that $\left(K_{6}, \odot_{3}, \mathbb{K}\right)$ define a sub-monoid. Tensor $\mathbb{K}=\mathbb{K}_{1}+\mathbb{K}_{2}$ is the unit element of $K_{6}$ for the composition $\odot_{3}$.

- Introducing $J_{6}$ the space of isotropic $6^{t h}$-order tensors given by $\mathbb{A}=a_{1} \mathbb{J}_{1}+$ $a_{2} \mathbb{J}_{2}+a_{3} \mathbb{J}_{3}+a_{4} \mathbb{J}_{4}$, it can be also shown from table 1 , that $\left(J_{6}, \odot_{3}, \mathbb{J}\right)$ define a sub-monoid. $\mathbb{J}=\mathbb{J}_{1}+\mathbb{J}_{4}$ is the unit element of $J_{6}$ for the composition $\odot_{3}$.

For a given $3^{r d}$-order tensor, the contractions $\mathbb{J}_{n} \odot_{3} \boldsymbol{a}$ provide four spherical tensors , which can be named partial spherical parts of $\boldsymbol{a}$ :

$$
\begin{aligned}
& \left(S_{1}(\boldsymbol{a})\right)_{i j k}=\frac{1}{5}\left(2 a_{p p k}-a_{k p p}\right) \delta_{i j}, \quad\left(S_{2}(\boldsymbol{a})\right)_{i j k}=\frac{1}{5}\left(3 a_{k p p}-a_{p p k}\right) \delta_{i j} \\
& \left(S_{3}(\boldsymbol{a})\right)_{i j k}=\frac{1}{10}\left(2 a_{p p i}-a_{i p p}\right) \delta_{j k}+\frac{1}{10}\left(2 a_{p p j}-a_{j p p}\right) \delta_{i k} \\
& \left(S_{4}(\boldsymbol{a})\right)_{i j k}=\frac{1}{10}\left(3 a_{i p p}-a_{p p i}\right) \delta_{j k}+\frac{1}{10}\left(3 a_{j p p}-a_{p p j}\right) \delta_{i k}
\end{aligned}
$$

Operators $S_{n}$ have the properties: $S_{n}\left(S_{n}(\boldsymbol{a})\right)=S_{n}(\boldsymbol{a})$ for $n=1$ and $n=4$ but $S_{n}\left(S_{n}(\boldsymbol{a})\right)=0$ for $n=2$ and $n=3$. In another hand, the deviatoric part of $\boldsymbol{a}$ can be decomposed into the partial deviatoric parts of $\boldsymbol{a}, D_{1}(\boldsymbol{a})$ and $D_{2}(\boldsymbol{a})$, which are defined by:

$$
\begin{aligned}
& \left(D_{1}(\boldsymbol{a})\right)_{i j k}=\frac{1}{3}\left(A_{i j k}+A_{i k j}+A_{j k i}\right)-\frac{1}{3}\left[\left(S_{1}(\boldsymbol{a})\right)_{i j k}+\left(S_{2}(\boldsymbol{a})\right)_{i j k}\right] \\
& -\frac{2}{3}\left[\left(S_{3}(\boldsymbol{a})\right)_{i j k}+\left(S_{4}(\boldsymbol{a})\right)_{i j k}\right] \\
& \left(D_{2}(\boldsymbol{a})\right)_{i j k}=\frac{1}{3}\left(2 A_{i j k}-A_{i k j}-A_{j k i}\right)-\frac{1}{3}\left[2\left(S_{1}(\boldsymbol{a})\right)_{i j k}+\left(S_{4}(\boldsymbol{a})\right)_{i j k}\right] \\
& +\frac{1}{3}\left[\left(S_{2}(\boldsymbol{a})\right)_{i j k}+\left(S_{3}(\boldsymbol{a})\right)_{i j k}\right]
\end{aligned}
$$

These partial spherical and deviatoric parts have the properties $D_{n}\left(S_{m}(\boldsymbol{a})\right)=$ $S_{m}\left(D_{n}(\boldsymbol{a})\right)=0$ whatever $n=1,2$ and $m=1,2,3,4$. As a consequence every $3^{r d}$-order tensor $\boldsymbol{a}$ can be decomposed into:

$$
\boldsymbol{a}=D_{1}(\boldsymbol{a})+D_{2}(\boldsymbol{a})+S_{1}(\boldsymbol{a})+S_{4}(\boldsymbol{a})
$$

Remark : A decomposition of a third order symmetric tensor (called SFH decomposition) has been introduced by Smyshlyaev \& Fleck (1996), formalized by Fleck \& Hutchinson (1997) and used more recently in the context of gradient plasticity by Gurtin \& Anand (2005). The SFH decomposition of a third order tensor reads: $\boldsymbol{a}=\boldsymbol{a}^{(1)}+\boldsymbol{a}^{(2)}+\boldsymbol{a}^{(3)}$ where the expressions of $\boldsymbol{a}^{(n)}$ for $n=1,2,3$ are recalled in 
appendix A. The concept of spherical and deviatoric part of a third order tensor has not been used by the authors. There are close relations with our approach because it can be shown that $a^{(1)}=D_{1}(\boldsymbol{a}), a^{(2)}=D_{2}(\boldsymbol{a})$ and $\boldsymbol{a}^{(3)}=S(\boldsymbol{a})$. However, the SFH decomposition uses only three terms whereas in the present study $\boldsymbol{a}$ is decomposed into four terms: two spherical parts and two deviatoric parts as shown in equation (2.10).

Similarly, a definition of the deviatoric part of a fourth order tensor has been proposed in Lubarda \& Krajcinovic (1993). However, the definition introduced by these authors can be used only for a tensor which is invariant by any permutation of its indices, while, in our paper, the considered fourth order tensor i symmetric only according to its two first ant two last indices. The $3^{r d}$-order tensor $\boldsymbol{b}=\mathbb{A} \odot_{3} \boldsymbol{a}$ can be decomposed into its partial spherical and deviatoric parts which are related to the ones of $\boldsymbol{a}$ by:

$$
\left\{\begin{array}{l}
D_{1}(\boldsymbol{b})=a_{1} D_{1}(\boldsymbol{a}) \\
D_{2}(\boldsymbol{b})=a_{2} D_{2}(\boldsymbol{a}) \\
S_{1}(\boldsymbol{b})=a_{3} S_{1}(\boldsymbol{a})+a_{4} S_{2}(\boldsymbol{a}) \\
S_{4}(\boldsymbol{b})=a_{5} S_{3}(\boldsymbol{a})+a_{6} S_{4}(\boldsymbol{a})
\end{array}\right.
$$

In which $a_{1} . . a_{6}$ are the components of $\mathbb{A}$ in the basis $\left(\mathbb{K}_{n}, \mathbb{J}_{m}\right)$ as defined in equation (2.7).

Consider two $6^{t h}$-order tensors $(\mathbb{A}, \mathbb{B}) \in E_{6}$. We denote by $a_{1}, . . a_{6}$ and $b_{1}, . . b_{6}$ their components within the basis $\left(\mathbb{K}_{n}, \mathbb{J}_{m}\right)$. The triple contraction between $\mathbb{A}$ and $\mathbb{B}$ leads to:

$$
\begin{aligned}
\mathbb{A} \odot \mathbb{B} & =a_{1} b_{1} \mathbb{K}_{1}+a_{2} b_{2} \mathbb{K}_{2}+\left(a_{3} b_{3}+a_{4} b_{5}\right) \mathbb{J}_{1}+\left(a_{3} b_{4}+a_{4} b_{6}\right) \mathbb{J}_{2} \\
& +\left(a_{5} b_{3}+a_{6} b_{5}\right) \mathbb{J}_{3}+\left(a_{5} b_{4}+a_{6} b_{6}\right) \mathbb{J}_{4}
\end{aligned}
$$

It is now possible to look for an inverse of $\mathbb{A} \in E_{6}$. Let $\mathbb{B} \in E_{6}$ be the inverse of $\mathbb{A}$ defined by $\mathbb{B} \odot_{3} \mathbb{A}=\mathbb{A} \odot_{3} \mathbb{B}=\mathbb{I}$. Note that $J_{6} \bigcap K_{6}=\{0\}$ and consequently the inverse of $\mathbb{A} \in E_{6}$ is the sum of $\mathbb{B}_{K}=\mathbb{K} \odot_{3} \mathbb{B}$, the inverse of $\mathbb{A}_{K}=\mathbb{K} \odot_{3} \mathbb{A} \in K_{6}$, and $\mathbb{B}_{J}=\mathbb{J} \odot_{3} \mathbb{B}$, the inverse of $\mathbb{A}_{J}=\mathbb{J} \odot_{3} \mathbb{A} \in J_{6}$. Finally, the components of $\mathbb{B}$ are given by:

$$
\mathbb{B}=\frac{1}{a_{1}} \mathbb{K}_{1}+\frac{1}{a_{2}} \mathbb{K}_{2}+\frac{1}{\Delta_{J}}\left[a_{6} \mathbb{J}_{1}-a_{4} \mathbb{J}_{2}+a_{5} \mathbb{J}_{3}-a_{3} \mathbb{J}_{4}\right]
$$

with: $\Delta_{J}=a_{3} a_{6}-a_{4} a_{5}$. As a consequence, the condition for $\mathbb{A}$ having an inverse is: $a_{1} a_{2} \Delta_{J} \neq 0$. The tensors which comply with this condition constitute a submonoid which has the properties of a group. The production of the inverse, if it exists, of any $6^{\text {th }}$-order isotropic tensor having minor symmetries is a clear advantage of the basis $\left(\mathbb{K}_{n}, \mathbb{J}_{m}\right)$, compared to the basis $\mathbb{T}_{n}$. The following section is devoted to the construction of a similar basis for $8^{\text {th }}$-order isotropic tensors.

\section{A basis for $8^{\text {th }}$-order isotropic tensors}

We now consider in (1.1) the case $n=4$. Consequently $\boldsymbol{a}$ and $\boldsymbol{b}$ are now two $4^{\text {th }}$-order tensors while $\mathbb{A}$ is a $8^{t h}$-order tensor. In the general case of an isotropic 
$8^{\text {th }}$-order tensor having no symmetries, it can be decomposed into a linear combination of 105 isotropic tensors whose components are obtained by the permutation according to indices $i, j, k, l, p, q, r, s$ of $\delta_{i j} \delta_{k l} \delta_{p q} \delta_{r s}$. In fact, 91 independent tensors are needed (Kearsley \& Fong 1975). Now, tensors $\mathbb{A}$ of components $A_{i j k l p q r s}$ are assumed to be symmetric according to indices $(i, j),(k, l),(p, q)$ and $(r, s)$ (called minor symmetries). So, among the 105 isotropic tensors quoted above, we can define 17 isotropic tensors having these four minor symmetries which are given by:

$$
\begin{array}{ll}
\left(\mathbb{T}_{1}\right)_{i j k l p q r s}= & \delta_{i j} \delta_{k l} \delta_{p q} \delta_{r s} \\
\left(\mathbb{T}_{2}\right)_{i j k l p q r s}= & \delta_{i j} \delta_{k l} I_{p q r s}, \quad\left(\mathbb{T}_{3}\right)_{i j k l p q r s}=\delta_{i j} \delta_{p q} I_{k l r s} \\
\left(\mathbb{T}_{4}\right)_{i j k l p q r s}=\delta_{i j} \delta_{r s} I_{k l p q}, \quad\left(\mathbb{T}_{5}\right)_{i j k l p q r s}=\delta_{k l} \delta_{p q} I_{i j r s} \\
\left(\mathbb{T}_{6}\right)_{i j k l p q r s}=\delta_{k l} \delta_{r s} I_{i j p q}, \quad\left(\mathbb{T}_{7}\right)_{i j k l p q r s}=\delta_{p q} \delta_{r s} I_{i j k l} \\
\left(\mathbb{T}_{8}\right)_{i j k l p q r s}=\delta_{i j} I_{k l p q r s}, \quad\left(\mathbb{T}_{9}\right)_{i j k l p q r s}=\delta_{k l} I_{i j p q r s} \\
\left(\mathbb{T}_{10}\right)_{i j k l p q r s}=\delta_{p q} I_{i j k l r s}, \quad\left(\mathbb{T}_{11}\right)_{i j k l p q r s}=\delta_{r s} I_{i j k l p q} \\
\left(\mathbb{T}_{12}\right)_{i j k l p q r s}=I_{i j k l} I_{p q r s}, \quad\left(\mathbb{T}_{13}\right)_{i j k l p q r s}=I_{i j p q} I_{k l r s} \\
\left(\mathbb{T}_{14}\right)_{i j k l p q r s}=I_{i j r s} I_{k l p q} \\
\left(\mathbb{T}_{15}\right)_{i j k l p q r s}=\frac{1}{4}\left(I_{i j p r} I_{k l q s}+I_{i j p s} I_{k l q r}+I_{i j q r} I_{k l p s}+I_{i j q s} I_{k l p r}\right) \\
\left(\mathbb{T}_{16}\right)_{i j k l p q r s}=\frac{1}{4}\left(I_{i j k r} I_{p q l s}+I_{i j k s} I_{p q l r}+I_{i j l r} I_{p q k s}+I_{i j l s} I_{p q k r}\right) \\
\left(\mathbb{T}_{17}\right)_{i j k l p q r s}=\frac{1}{4}\left(I_{i j k p} I_{r s l q}+I_{i j k q} I_{r s l p}+I_{i j l p} I_{r s k q}+I_{i j l q} I_{r s k p}\right)
\end{array}
$$

Note that a more refined analysis of the 17 tensors defined above show that they do not constitute an irreducible basis for $8^{t h}$-order tensor. More precisely, those tensors comply with the following relation:

$$
\begin{aligned}
& -\mathbb{T}_{1}+\mathbb{T}_{2}+\mathbb{T}_{3}+\mathbb{T}_{4}+\mathbb{T}_{5}+\mathbb{T}_{6}+\mathbb{T}_{7}-2\left(\mathbb{T}_{8}+\mathbb{T}_{9}+\mathbb{T}_{10}+\mathbb{T}_{11}\right) \\
& -\left(\mathbb{T}_{12}+\mathbb{T}_{13}+\mathbb{T}_{14}\right)+2\left(\mathbb{T}_{15}+\mathbb{T}_{16}+\mathbb{T}_{17}\right)=0
\end{aligned}
$$

All $8^{\text {th }}$-order isotropic tensor having the minor symmetries is defined by 16 independent coefficients and then can be decomposed as a linear combination of 16 tensors chosen among those of (3.1). As for the case of a $6^{\text {th }}$-order tensor, a basis made up of tensors $\mathbb{T}_{n}$ is not useful for doing the classical tensorial operations and specially the inversion since $\mathbb{T}_{n} \odot \mathbb{T}_{m} \neq 0$ whatever $n, m=1 . .17$.

The methodology used is the same as the one applied through the previous section. The first step consists in splitting a $4^{t h}$-order tensor $\boldsymbol{a}$ into its deviatoric and spherical parts:

$$
\boldsymbol{a}=D(\boldsymbol{a})+S(\boldsymbol{a})
$$


where $D(\boldsymbol{a})$ is the deviatoric part of $\boldsymbol{a}$ such that: $[D(\boldsymbol{a})]_{i j p p}=[D(\boldsymbol{a})]_{p p i j}=$ $[D(\boldsymbol{a})]_{i p j p}=0$. Now, for a $4^{\text {th }}$-order tensor, it is possible to find tensors for which the contraction over two indices, which defines the deviatoric part, is not zero, but for which the double contraction over indices is null. Let us call first spherical part $S^{1}(\boldsymbol{a})$ such tensors, which comply to $\left[S^{1}(\boldsymbol{a})\right]_{p p q q}=\left[S^{1}(\boldsymbol{a})\right]_{p q p q}=0$. Now, $\boldsymbol{a}$ can be decomposed as:

$$
\boldsymbol{a}=D(\boldsymbol{a})+S^{1}(\boldsymbol{a})+S^{2}(\boldsymbol{a})
$$

where the second spherical part $S^{2}(\boldsymbol{a})=S(\boldsymbol{a})-S^{1}(\boldsymbol{a})$ has been introduced. The components of $D(\boldsymbol{a}), S^{1}(\boldsymbol{a})$ and $S^{2}(\boldsymbol{a})$ are given by:

$$
\begin{aligned}
& {\left[S^{2}(\boldsymbol{a})\right]_{i j k l}=} \frac{1}{15}\left(2 a_{p p q q}-a_{p q p q}\right) \delta_{i j} \delta_{k l}+\frac{1}{15}\left(3 a_{p q p q}-a_{p p q q}\right) I_{i j k l} \\
& {\left[S^{1}(\boldsymbol{a})\right]_{i j k l}=} \frac{5}{7}\left(\alpha_{i j} \delta_{k l}+\alpha_{k l} \delta_{i j}\right)-\frac{4}{7}\left(\beta_{i j} \delta_{k l}+\beta_{k l} \delta_{i j}\right) \\
&-\frac{2}{7}\left(\alpha_{i k} \delta_{j l}+\alpha_{i l} \delta_{j k}+\alpha_{j k} \delta_{i l}+\alpha_{j l} \delta_{i k}\right) \\
&+\frac{3}{7}\left(\beta_{i k} \delta_{j l}+\beta_{i l} \delta_{j k}+\beta_{j k} \delta_{i l}+\beta_{j l} \delta_{i k}\right) \\
&+\frac{1}{5}\left(\eta_{i k} \delta_{j l}+\eta_{i l} \delta_{j k}+\eta_{j k} \delta_{i l}+\eta_{j l} \delta_{i k}\right) \\
&+\frac{1}{3}\left(\gamma_{i j} \delta_{k l}-\gamma_{k l} \delta_{i j}\right) \\
& D(\boldsymbol{a})=\boldsymbol{a}-S^{1}(\boldsymbol{a})-S^{2}(\boldsymbol{a})
\end{aligned}
$$

with:

$$
\begin{aligned}
\alpha_{i j} & =\frac{1}{2}\left(a_{i j p p}+a_{p p i j}\right)-\frac{1}{3} a_{p p q q} \delta_{i j} \\
\beta_{i j} & =\frac{1}{2}\left(a_{i p j p}+a_{j p i p}\right)-\frac{1}{3} a_{p q p q} \delta_{i j} \\
\gamma_{i j} & =\frac{1}{2}\left(a_{i j p p}-a_{p p i j}\right), \quad \eta_{i j}=\frac{1}{2}\left(a_{i p j p}-a_{j p i p}\right)
\end{aligned}
$$

$\boldsymbol{\alpha}, \boldsymbol{\beta}, \boldsymbol{\gamma}$ and $\boldsymbol{\eta}$ are traceless. $S^{1}(\boldsymbol{a})$ and $S^{2}(\boldsymbol{a})$ have the properties:

$$
\begin{aligned}
& {\left[S^{2}(\boldsymbol{a})\right]_{p p q q}=a_{p p q q}, \quad\left[S^{2}(\boldsymbol{a})\right]_{p q p q}=a_{p q p q}} \\
& {\left[S^{1}(\boldsymbol{a})+S^{2}(\boldsymbol{a})\right]_{i j p p}=a_{i j p p}, \quad\left[S^{1}(\boldsymbol{a})+S^{2}(\boldsymbol{a})\right]_{p p i j}=a_{p p i j}} \\
& {\left[S^{1}(\boldsymbol{a})+S^{2}(\boldsymbol{a})\right]_{i p j p}=a_{i p j p}}
\end{aligned}
$$

We introduce $\mathbb{J}^{1}, \mathbb{J}^{2}, \mathbb{K}$, and $\mathbb{I}$ such that $S^{1}(\boldsymbol{a})=\mathbb{J}^{1} \odot_{4} \boldsymbol{a}, S^{2}(\boldsymbol{a})=\mathbb{J}^{2} \odot_{4} \boldsymbol{a}$, $D(\boldsymbol{a})=\mathbb{K} \odot_{4} \boldsymbol{a}$ and $\boldsymbol{a}=\mathbb{I} \odot_{4} \boldsymbol{a}$. These tensors can be expressed in the basis $\mathbb{T}_{n}$ as 
follows:

$$
\begin{aligned}
& \mathbb{J}^{2}=\frac{1}{15}\left(2 \mathbb{T}_{1}-\mathbb{T}_{2}-\mathbb{T}_{7}+3 \mathbb{T}_{12}\right) \\
& \mathbb{J}^{1}=\frac{1}{21}\left(-10 \mathbb{T}_{1}+8 \mathbb{T}_{2}+11 \mathbb{T}_{3}+4 \mathbb{T}_{4}+4 \mathbb{T}_{5}+11 \mathbb{T}_{6}+8 \mathbb{T}_{7}\right) \\
& \quad-\frac{2}{7}\left(2 \mathbb{T}_{8}+2 \mathbb{T}_{9}+2 \mathbb{T}_{10}+2 \mathbb{T}_{11}-3 \mathbb{T}_{16}-3 \mathbb{T}_{17}\right) \\
& \mathbb{I}=\mathbb{T}_{13}, \quad \mathbb{K}=\mathbb{I}-\mathbb{J}^{1}-\mathbb{J}^{2}
\end{aligned}
$$

Let us decompose $\mathbb{A} \in E_{8}$ as follows: $\mathbb{A}=\mathbb{A}_{J^{2}}+\mathbb{A}_{J^{1}}+\mathbb{A}_{K}$ where $\mathbb{A}_{J^{2}}=\mathbb{J}^{2} \odot_{4} \mathbb{A}$, $\mathbb{A}_{J^{1}}=\mathbb{J}^{1} \odot_{4} \mathbb{A}$ and $\mathbb{A}_{K}=\mathbb{K} \odot_{4} \mathbb{A}$. As for the case of a $6^{t h}$-order, tensor $\mathbb{A}_{J^{2}}$ is defined by 4 independent coefficients. This suggests that there exist four tensors $\mathbb{J}_{n}^{2}$ for $n=1,2,3,4$ such that $\mathbb{A}_{J^{2}}=a_{1} \mathbb{J}_{1}^{2}+a_{2} \mathbb{J}_{2}^{2}+a_{3} \mathbb{J}_{3}^{2}+a_{4} \mathbb{J}_{4}^{2}, \mathbb{J}^{1} \odot_{4} \mathbb{J}_{n}^{2}=\mathbb{J}_{n}^{2} \odot_{4} \mathbb{J}^{1}=$ $\mathbb{J}_{n}^{2} \odot_{4} \mathbb{K}=\mathbb{J}_{n}^{2} \odot_{4} \mathbb{K}=0$, and $\mathbb{J}_{n}^{2} \odot_{4} \mathbb{J}^{2}=\mathbb{J}^{2} \odot_{4} \mathbb{J}_{n}^{2}=\mathbb{J}_{n}^{2}$ whatever the value of $n=1,2,3,4$. These tensors read:

$$
\begin{aligned}
\mathbb{J}_{1}^{2}=\frac{1}{15}\left(2 \mathbb{T}_{1}-\mathbb{T}_{2}\right), & \mathbb{J}_{2}^{2}=\frac{1}{15}\left(3 \mathbb{T}_{2}-\mathbb{T}_{1}\right) \\
\mathbb{J}_{3}^{2}=\frac{1}{15}\left(2 \mathbb{T}_{7}-\mathbb{T}_{12}\right), & \mathbb{J}_{4}^{2}=\frac{1}{15}\left(3 \mathbb{T}_{12}-\mathbb{T}_{7}\right)
\end{aligned}
$$

Note that $\mathbb{J}^{2}=\mathbb{J}_{1}^{2}+\mathbb{J}_{4}^{2}$. The quadruple contraction between the different tensors $\mathbb{J}_{n}^{2}$ for $n=1,2,3,4$ are given in table 3 :

\begin{tabular}{|c|c|c|c|c|}
\hline$\odot_{4}$ & $\mathbb{J}_{1}^{2}$ & $\mathbb{J}_{2}^{2}$ & $\mathbb{J}_{3}^{2}$ & $\mathbb{J}_{4}^{2}$ \\
\hline $\mathbb{J}_{1}^{2}$ & $\mathbb{J}_{1}^{2}$ & $\mathbb{J}_{2}^{2}$ & 0 & 0 \\
\hline $\mathbb{J}_{2}^{2}$ & 0 & 0 & $\mathbb{J}_{1}^{2}$ & $\mathbb{J}_{2}^{2}$ \\
\hline $\mathbb{J}_{3}^{2}$ & $\mathbb{J}_{3}^{2}$ & $\mathbb{J}_{4}^{2}$ & 0 & 0 \\
\hline $\mathbb{J}_{4}^{2}$ & 0 & 0 & $\mathbb{J}_{3}^{2}$ & $\mathbb{J}_{4}^{2}$ \\
\hline
\end{tabular}

Table 3 : The quadruple contraction between the $\mathbb{J}_{n}^{1}$ for $n=1 . .10$

It can be observed that the structure of the composition of all $\mathbb{J}_{n}^{2}$ has the same properties as the one obtained for $\mathbb{J}_{n}$ in the last section.

Now it is possible to show that tensors $\mathbb{A}_{J^{1}}$ constitute a vector space having dimension 8. Therefore $\mathbb{A}_{J^{1}}$ has 10 independent coefficients, which suggests the existence of 10 tensors $\mathbb{J}_{n}^{2}$ such that $\mathbb{A}_{J^{1}}=a_{1} \mathbb{J}_{1}^{1}+\ldots+a_{10} \mathbb{J}_{10}^{1}, \mathbb{J}^{2} \odot_{4} \mathbb{J}_{n}^{1}=\mathbb{J}_{n}^{1} \odot_{4} \mathbb{J}^{2}=\mathbb{J}_{n}^{1} \odot_{4} \mathbb{K}=$ $\mathbb{J}_{n}^{1} \odot_{4} \mathbb{K}=0$, and $\mathbb{J}_{n}^{1} \odot_{4} \mathbb{J}^{1}=\mathbb{J}^{1} \odot_{4} \mathbb{J}_{n}^{1}=\mathbb{J}_{n}^{1}$ whatever the value of $n=1 . .10$. These 
tensors read:

$$
\begin{aligned}
\mathbb{J}_{1}^{1}= & \frac{1}{3} \mathbb{T}_{3}-\frac{1}{9} \mathbb{T}_{1}, \quad \mathbb{J}_{2}^{1}=\frac{1}{3} \mathbb{T}_{4}-\frac{1}{9} \mathbb{T}_{1} \\
\mathbb{J}_{3}^{1}= & \mathbb{T}_{8}+\frac{2}{9} \mathbb{T}_{1}-\frac{1}{3}\left(\mathbb{T}_{2}+\mathbb{T}_{3}+\mathbb{T}_{4}\right) \\
\mathbb{J}_{4}^{1}= & \frac{1}{3} \mathbb{T}_{5}-\frac{1}{9} \mathbb{T}_{1}, \quad \mathbb{J}_{5}^{1}=\frac{1}{3} \mathbb{T}_{6}-\frac{1}{9} \mathbb{T}_{1} \\
\mathbb{J}_{6}^{1}= & \mathbb{T}_{9}+\frac{2}{9} \mathbb{T}_{1}-\frac{1}{3}\left(\mathbb{T}_{2}+\mathbb{T}_{5}+\mathbb{T}_{6}\right) \\
\mathbb{J}_{7}^{1}= & \frac{4}{7}\left[\mathbb{T}_{10}+\frac{2}{9} \mathbb{T}_{1}-\frac{1}{3}\left(\mathbb{T}_{3}+\mathbb{T}_{5}+\mathbb{T}_{7}\right)\right] \\
\mathbb{J}_{8}^{1}= & \frac{4}{7}\left[\mathbb{T}_{11}+\frac{2}{9} \mathbb{T}_{1}-\frac{1}{3}\left(\mathbb{T}_{4}+\mathbb{T}_{6}+\mathbb{T}_{7}\right)\right] \\
\mathbb{J}_{9}^{1}= & \frac{1}{7}\left[\frac{11}{9} \mathbb{T}_{1}-\frac{1}{3}\left(\mathbb{T}_{2}+\mathbb{T}_{7}\right)-\frac{5}{3}\left(\mathbb{T}_{3}+\mathbb{T}_{4}+\mathbb{T}_{5}+\mathbb{T}_{6}\right)\right. \\
& \left.+2\left(\mathbb{T}_{8}+\mathbb{T}_{9}+\mathbb{T}_{10}+\mathbb{T}_{11}\right)-\mathbb{T}_{12}+3\left(\mathbb{T}_{13}+\mathbb{T}_{14}\right)-6 \mathbb{T}_{15}\right] \\
\mathbb{J}_{10}^{1}= & \frac{2}{5}\left(\mathbb{T}_{17}-\mathbb{T}_{16}\right)
\end{aligned}
$$

\begin{tabular}{|c|c|c|c|c|c|c|c|c|c|c|}
\hline$\odot_{4}$ & $\mathbb{J}_{1}^{1}$ & $\mathbb{J}_{2}^{1}$ & $\mathbb{J}_{3}^{1}$ & $\mathbb{J}_{4}^{1}$ & $\mathbb{J}_{5}^{1}$ & $\mathbb{J}_{6}^{1}$ & $\mathbb{J}_{7}^{1}$ & $\mathbb{J}_{8}^{1}$ & $\mathbb{J}_{9}^{1}$ & $\mathbb{J}_{10}^{1}$ \\
\hline $\mathbb{J}_{1}^{1}$ & $\mathbb{J}_{1}^{1}$ & $\mathbb{J}_{2}^{1}$ & $\mathbb{J}_{3}^{1}$ & 0 & 0 & 0 & 0 & 0 & 0 & 0 \\
\hline $\mathbb{J}_{2}^{1}$ & 0 & 0 & 0 & $\mathbb{J}_{1}^{1}$ & $\mathbb{J}_{2}^{1}$ & $\mathbb{J}_{3}^{1}$ & 0 & 0 & 0 & 0 \\
\hline $\mathbb{J}_{3}^{1}$ & 0 & 0 & 0 & 0 & 0 & 0 & $\mathbb{J}_{1}^{1}$ & $\mathbb{J}_{2}^{1}$ & $\mathbb{J}_{3}^{1}$ & 0 \\
\hline $\mathbb{J}_{4}^{1}$ & $\mathbb{J}_{4}^{1}$ & $\mathbb{J}_{5}^{1}$ & $\mathbb{J}_{6}^{1}$ & 0 & 0 & 0 & 0 & 0 & 0 & 0 \\
\hline $\mathbb{J}_{5}^{1}$ & 0 & 0 & 0 & $\mathbb{J}_{4}^{1}$ & $\mathbb{J}_{5}^{1}$ & $\mathbb{J}_{6}^{1}$ & 0 & 0 & 0 & 0 \\
\hline $\mathbb{J}_{6}^{1}$ & 0 & 0 & 0 & 0 & 0 & 0 & $\mathbb{J}_{4}^{1}$ & $\mathbb{J}_{5}^{1}$ & $\mathbb{J}_{6}^{1}$ & 0 \\
\hline $\mathbb{J}_{7}^{1}$ & $\mathbb{J}_{7}^{1}$ & $\mathbb{J}_{8}^{1}$ & $\mathbb{J}_{9}^{1}$ & 0 & 0 & 0 & 0 & 0 & 0 & 0 \\
\hline $\mathbb{J}_{8}^{1}$ & 0 & 0 & 0 & $\mathbb{J}_{7}^{1}$ & $\mathbb{J}_{8}^{1}$ & $\mathbb{J}_{9}^{1}$ & 0 & 0 & 0 & 0 \\
\hline $\mathbb{J}_{9}^{1}$ & 0 & 0 & 0 & 0 & 0 & 0 & $\mathbb{J}_{7}^{1}$ & $\mathbb{J}_{8}^{1}$ & $\mathbb{J}_{9}^{1}$ & 0 \\
\hline $\mathbb{J}_{10}^{1}$ & 0 & 0 & 0 & 0 & 0 & 0 & 0 & 0 & 0 & $\mathbb{J}_{10}^{1}$ \\
\hline
\end{tabular}

The contraction between the different tensors $\mathbb{J}_{n}^{1}$ for $n=1 . .10$ are given below:

Table 4: The quadruple contraction between the $\mathbb{J}_{n}^{1}$ for $n=1 . .10$

Note that $\mathbb{J}^{1}=\mathbb{J}_{1}^{1}+\mathbb{J}_{5}^{1}+\mathbb{J}_{9}^{1}+\mathbb{J}_{10}^{1}$.

Finally, $\mathbb{A}_{K}$ is within a vector space having dimension 2 . So we introduce $\mathbb{K}_{n}$ such that $\mathbb{A}_{K}=a_{1} \mathbb{K}_{1}+a_{2} \mathbb{K}_{2}, \mathbb{J}^{p} \odot_{4} \mathbb{K}_{n}=\mathbb{K}_{n} \odot_{4} \mathbb{J}^{p}=0$, and $\mathbb{K}_{n} \odot_{4} \mathbb{K}=\mathbb{K} \odot_{4} \mathbb{K}_{n}=\mathbb{K}_{n}$ 
whatever the value of $n=1 . .2$. These tensors read:

$$
\begin{aligned}
\mathbb{K}_{1}= & \frac{1}{6}\left(\mathbb{T}_{4}+\mathbb{T}_{5}-\mathbb{T}_{3}-\mathbb{T}_{6}\right)+\frac{1}{2}\left(\mathbb{T}_{13}-\mathbb{T}_{14}\right)+\frac{2}{5}\left(\mathbb{T}_{16}-\mathbb{T}_{17}\right) \\
\mathbb{K}_{2}= & \frac{1}{35}\left(12 \mathbb{T}_{1}-11 \mathbb{T}_{2}-11 \mathbb{T}_{7}+13 \mathbb{T}_{12}\right)-\frac{5}{14}\left(\mathbb{T}_{3}+\mathbb{T}_{4}+\mathbb{T}_{5}+\mathbb{T}_{6}\right) \\
& +\frac{4}{7}\left(\mathbb{T}_{8}+\mathbb{T}_{9}+\mathbb{T}_{10}+\mathbb{T}_{11}\right)+\frac{1}{2}\left(\mathbb{T}_{13}+\mathbb{T}_{14}\right)-\frac{6}{7}\left(\mathbb{T}_{16}+\mathbb{T}_{17}\right)
\end{aligned}
$$

Note that $\mathbb{K}=\mathbb{K}_{1}+\mathbb{K}_{2}$.

The contraction between the different tensors $\mathbb{K}_{n}$ for $n=1 . .2$ are given below:

\begin{tabular}{|c|c|c|}
\hline$\odot_{4}$ & $\mathbb{K}_{1}$ & $\mathbb{K}_{2}$ \\
\hline $\mathbb{K}_{1}$ & $\mathbb{K}_{1}$ & 0 \\
\hline $\mathbb{K}_{2}$ & 0 & $\mathbb{K}_{2}$ \\
\hline
\end{tabular}

Table 5: The quadruple contraction between the $\mathbb{K}_{n}$ for $n=1 . .2$

Note that the table of products is the same as for the case of $6^{\text {th }}$-order tensors. All $8^{\text {th }}$-order tensors $\mathbb{A}$ having the minor symmetries can be decomposed by using the irreducible basis $\left(\mathbb{J}_{n}^{1}, \mathbb{J}_{m}^{2}, \mathbb{K}_{p}\right)$. Appendix B produces the relations allowing to obtain the components of any $8^{t h}$-order tensor within basis $\left(\mathbb{J}_{n}^{1}, \mathbb{J}_{m}^{2}, \mathbb{K}_{p}\right)$ from its components in the basis $\left.\mathbb{T}_{i}\right)$. As for the case of a $6^{t h}$-order tensor, $\left(E_{4}, \odot_{4}, \mathbb{I}\right)$ define a monoid for the composition $\odot_{4}$, the unit tensor for $\odot_{4}$ being $\mathbb{I}=\mathbb{T}_{13}$.

The following decomposition of the space $E_{8}=J_{8}^{1} \cup J_{8}^{2} \bigcup K_{8}$ is used where $J_{8}^{2}$ define the sub-space of isotropic $8^{\text {th }}$-order tensors which can be decomposed in the basis of tensors $\mathbb{J}_{n}^{2}$ for $n=1 . .4$, the sub-space $J_{8}^{1}$ and $K_{8}$ being respectively associated to $\mathbb{J}_{n}^{1}$ for $n=1 . .10$ and $\mathbb{K}_{n}$ for $n=1 . .2$. It can be observed that $\left(K_{8}, \odot_{4}, \mathbb{K}\right)$ define a commutative sub-monoid, while $\left(J_{8}^{2}, \odot_{4}, \mathbb{J}^{2}\right)$ and $\left(J_{8}^{1}, \odot_{4}, \mathbb{J}^{1}\right)$ define two sub-monoids.

Let us decompose $\mathbb{A} \in E_{8}$ by using the new basis:

$$
\begin{aligned}
& \mathbb{A}=a_{1} \mathbb{K}_{1}+a_{2} \mathbb{K}_{2}+a_{4} \mathbb{J}_{1}^{1}+a_{5} \mathbb{J}_{2}^{1}+a_{6} \mathbb{J}_{3}^{1}+a_{7} \mathbb{J}_{4}^{1}+a_{8} \mathbb{J}_{5}^{1}+a_{9} \mathbb{J}_{6}^{1} \\
& +a_{10} \mathbb{J}_{7}^{1}+a_{11} \mathbb{J}_{8}^{1}+a_{12} \mathbb{J}_{9}^{1}+a_{13} \mathbb{J}_{10}^{1}+a_{13} \mathbb{J}_{1}^{2}+a_{14} \mathbb{J}_{2}^{2}+a_{15} \mathbb{J}_{3}^{2}+a_{16} \mathbb{J}_{4}^{2}
\end{aligned}
$$

Let us do the same with a second tensor $\mathbb{B}$, its components within the new basis being denoted by $b_{i}$ for $i=1 . .16$. Defining now $\mathbb{C}$ by $\mathbb{C}=\mathbb{A} \odot{ }_{4} \mathbb{B}$, its components 
within the new basis are given by:

$$
\begin{aligned}
& c_{1}=a_{1} b_{1}, \quad c_{2}=a_{2} b_{2} \\
& c_{3}=a_{3} b_{3}+a_{4} b_{6}+a_{5} b_{9}, \quad c_{4}=a_{3} b_{4}+a_{4} b_{7}+a_{5} b_{10} \\
& c_{5}=a_{3} b_{5}+a_{4} b_{8}+a_{5} b_{11}, \quad c_{6}=a_{6} b_{3}+a_{7} b_{6}+a_{8} b_{9} \\
& c_{7}=a_{6} b_{4}+a_{7} b_{7}+a_{8} b_{10}, \quad c_{8}=a_{6} b_{5}+a_{7} b_{8}+a_{8} b_{11} \\
& c_{9}=a_{9} b_{3}+a_{10} b_{6}+a_{11} b_{9}, \quad c_{10}=a_{9} b_{4}+a_{10} b_{7}+a_{11} b_{10} \\
& c_{11}=a_{9} b_{5}+a_{10} b_{8}+a_{11} b_{11}, \quad c_{12}=a_{12} b_{12} \\
& c_{13}=a_{13} b_{13}+a_{14} b_{15}, \quad c_{14}=a_{13} b_{14}+a_{14} b_{16} \\
& c_{15}=a_{15} b_{13}+a_{16} b_{15}, \quad c_{16}=a_{15} b_{14}+a_{16} b_{16}
\end{aligned}
$$

We now look for the inverse of a $8^{\text {th }}$-order tensor $\mathbb{A}$. The components of $\mathbb{B}$, solution of the equations $\mathbb{B} \odot_{4} \mathbb{A}=\mathbb{A} \odot_{4} \mathbb{B}=\mathbb{I}$ are:

$$
\begin{aligned}
& b_{1}=\frac{1}{a_{1}}, \quad b_{2}=\frac{1}{a_{2}} \\
& b_{3}=\frac{a_{7} a_{11}-a_{8} a_{10}}{\Delta_{J^{1}}}, \quad b_{4}=\frac{a_{5} a_{10}-a_{4} a_{11}}{\Delta_{J^{1}}}, \quad b_{5}=\frac{a_{4} a_{8}-a_{5} a_{7}}{\Delta_{J^{1}}} \\
& b_{6}=\frac{a_{8} a_{9}-a_{6} a_{11}}{\Delta_{J^{1}}}, \quad b_{7}=\frac{a_{3} a_{11}-a_{5} a_{9}}{\Delta_{J^{1}}}, \quad b_{9}=\frac{a_{5} a_{6}-a_{3} a_{8}}{\Delta_{J^{1}}} \\
& b_{9}=\frac{a_{6} a_{10}-a_{7} a_{9}}{\Delta_{J^{1}}}, \quad b_{10}=\frac{a_{4} a_{9}-a_{3} a_{10}}{\Delta_{J^{1}}}, \quad b_{11}=\frac{a_{3} a_{7}-a_{4} a_{6}}{\Delta_{J^{1}}} \\
& b_{12}=\frac{1}{a_{12}}, \quad b_{13}=\frac{a_{16}}{\Delta_{J^{2}}}, \quad b_{14}=-\frac{a_{14}}{\Delta_{J^{2}}}, \quad b_{15}=-\frac{a_{15}}{\Delta_{J^{2}}}, \quad b_{16}=\frac{a_{13}}{\Delta_{J^{2}}}
\end{aligned}
$$

with:

$$
\begin{aligned}
& \Delta_{J^{2}}=a_{13} a_{16}-a_{14} a_{15} \\
& \Delta_{J^{1}}=a_{3} a_{7} a_{11}+a_{4} a_{8} a_{9}+a_{5} a_{6} a_{10}-a_{3} a_{8} a_{10}-a_{4} a_{6} a_{11}-a_{5} a_{7} a_{9}
\end{aligned}
$$

The condition for $\mathbb{A}$ having an inverse is: $a_{1} a_{2} a_{12} \Delta_{J^{1}} \Delta_{J^{2}} \neq 0$

\section{The case of higher order isotropic tensors}

We aim at generalizing the methodology proposed in the previous sections to the case of $2 n^{t h}$-order tensor for $n \geq 5$. To this aim, consider a $n^{\text {th }}$ order tensor $\boldsymbol{a}$, its components being denoted by $a_{i j k . . l}$. This tensor is assumed symmetric according to its two first indices $a_{i j k . . l}=a_{j i k . . l}$ and also according to its $n-2$ last indices $k . . l$. Tensor $\boldsymbol{a}$ can be decomposed as follows:

$$
\boldsymbol{a}=D(\boldsymbol{a})+\left[S^{1}(\boldsymbol{a})+S^{2}(\boldsymbol{a}) \ldots+S^{p}(\boldsymbol{a})\right]
$$

with $n=2 p$ if $n$ is an even number but $n=2 p+1$ if $n$ is an odd number. $D(\boldsymbol{a})$ is the deviatoric part of $\boldsymbol{a}$ such that $[D(\boldsymbol{a})]_{\text {ppk..l }}=[D(\boldsymbol{a})]_{i j p p k . . l}=[D(\boldsymbol{a})]_{i p j p k . . l}=0$. 
In the expression above, $S^{p}(\boldsymbol{a})$ denotes $n^{t h}$ spherical part of $p$. Tensors $S^{p}(\boldsymbol{a})$ have the properties:

$$
\begin{aligned}
& {\left[S^{1}(\boldsymbol{a})+S^{2}(\boldsymbol{a}) \ldots+S^{p}(\boldsymbol{a})\right]_{i j p p k . . l}=a_{i j p p k . . l}} \\
& {\left[S^{1}(\boldsymbol{a})+S^{2}(\boldsymbol{a}) \ldots+S^{p}(\boldsymbol{a})\right]_{p p i j k . . l}=a_{p p i j k . . l}} \\
& {\left[S^{1}(\boldsymbol{a})+S^{2}(\boldsymbol{a}) \ldots+S^{p}(\boldsymbol{a})\right]_{i p j p k . . l}=a_{i p j p k . . l}} \\
& {\left[S^{2}(\boldsymbol{a}) \ldots+S^{p}(\boldsymbol{a})\right]_{i j p p q q k . . l}=a_{i j p p q q k . . l}} \\
& {\left[S^{2}(\boldsymbol{a}) \ldots+S^{p}(\boldsymbol{a})\right]_{\text {ppijqqk..l }}=a_{\text {ppijqqk..l}}} \\
& {\left[S^{2}(\boldsymbol{a}) \ldots+S^{p}(\boldsymbol{a})\right]_{i p j p q q k . . l}=a_{i p j p q q k . . l}} \\
& \text { etc... }
\end{aligned}
$$

Consequently, we introduce tensors $\mathbb{K}, \mathbb{J}^{p}$ for $p=1,2,3 \ldots$ and $\mathbb{I}$ such that: $D(\boldsymbol{a})=$ $\mathbb{K} \odot_{n} \boldsymbol{a}, S^{p}(\boldsymbol{a})=\mathbb{J}^{p} \odot_{n} \boldsymbol{a}$ and $\mathbb{I} \odot_{n} \boldsymbol{a}=\boldsymbol{a}$. We can define the independent subspaces $K_{2 n}, J_{2 n}^{p}$ for $p=1,2,3$.. associated to $\mathbb{K}, \mathbb{J}^{p}$, used for the decomposition of $\mathbb{A}$. The second step is to apply the decomposition $\mathbb{A}_{K}=a_{1} \mathbb{K}_{1}+a_{2} \mathbb{K}_{2}+\ldots$, $\mathbb{A}_{J^{p}}=a_{1} \mathbb{J}_{1}^{p}+a_{2} \mathbb{J}_{2}^{p}+\ldots$. Consequently, $\mathbb{A}$, is decomposed as:

$$
\mathbb{A}=\sum_{n} a_{n} \mathbb{K}_{n}+\sum_{p} \sum_{n} a_{p m} \rrbracket_{n}^{p}
$$

In table 6 are given the number of irreducible tensors $\mathbb{K}_{n}, \mathbb{J}_{n}^{p}$ for $2 \leq n \leq 6$.

\begin{tabular}{c|c|c|c|c|cc} 
& $K_{2 n}$ & $J_{2 n}^{1}$ & $J_{2 n}^{2}$ & $J_{2 n}^{3}$ & $J_{2 n}^{4}$ & $\ldots$ \\
\hline $2 n=4$ & 1 & 1 & 0 & 0 & 0 & \\
\hline $2 n=6$ & 2 & 4 & 0 & 0 & 0 & \\
\hline $2 n=8$ & 2 & 10 & 4 & 0 & 0 & \\
\hline $2 n=10$ & 3 & 13 & 9 & 0 & 0 \\
\hline $2 n=12$ & 2 & 13 & 17 & 4 & 0
\end{tabular}

Table 6: Number of irreducible elements of the sub-space $K_{2 n}$ and $J_{2 n}^{p}$

\section{Higher order inhomogeneity problem: the spherical inclusion in an infinite matrix subjected to a polynomial remote strain field}

The Eshelby's "inhomogeneity problem" (Eshelby 1957) is well known for the case of a given constant strain field at infinity: it gives the strain field inside an ellipsoidal inclusion having elastic properties which are different from the material outside 
the inclusion. This problem uses the solution of the "inclusion problem" for which a constant free deformation is given within an ellipsoidal part of an homogeneous material. The inclusion problem can be extended to the case of free deformations which have a polynomial form, but the solution of the "inhomogeneity problem" for the case of polynomial strain fields at infinity needs the inversion of higher order tensors. In this section, the method used for obtaining the inverse of higher order tensors is used for solving the "inhomogeneity problem" in the case of spherical inhomogeneities made up of an isotropic material and located within an infinite isotropic medium. Let us consider a spherical inclusion located at $x_{i}=0$ made up of an isotropic elastic material of rigidity $C_{i j k l}$ embedded in an infinite isotropic elastic matrix whose rigidity is $C_{i j k l}^{0}$. We denote by $\lambda, \mu, \nu\left(\operatorname{resp} . \lambda_{0}, \mu_{0}, \nu_{0}\right)$ the Lamé moduli and the Poisson ratio of the inclusion (respectively of the matrix). The inclusion is subjected to a polynomial remote strain field $\varepsilon^{\infty}(x)=e_{i j}+e_{i j k} x_{k}+$ $e_{i j k l} x_{k} x_{l}$. It has been proved (see Mura 1987 in the case of an infinite isotropic medium and the work of Asaro \& Barnett 1975 in the anisotropic context) that the strain field within the inclusion is also a polynomial and reads:

$$
a_{i j}(x)=a_{i j}+a_{i j k} x_{k}+a_{i j k l} x_{k} x_{l} \ldots
$$

In the following a series for $a_{i j}(x)$ at the second order is considered, $a_{i j}, a_{i j k}$ and $a_{i j k l}$ are solutions of:

$$
\begin{aligned}
& e_{i j}=\left[I_{i j p q}-P_{i j m n}^{0} \delta C_{m n p q}\right] a_{p q}+c_{i j} \\
& e_{i j k}=\left[I_{i j p q} \delta_{k r}-P_{i j k m n r}^{0} \delta C_{m n p q}\right] a_{p q r} \\
& e_{i j k l}=\left[I_{i j p q} I_{k l r s}-P_{i j k l m n r s}^{0} \delta C_{m n p q}\right] a_{p q r s} \\
& \text { etc... }
\end{aligned}
$$

with:

$$
c_{i j}=Q_{i j m n r s}^{0} \delta C_{m n p q} a_{p q r s}
$$

In the expression above $\delta C_{i j k l}=C_{i j k l}-C_{i j k l}^{0}$ and $P_{i j m n}^{0}$ are the components of the Hill tensor (Hill 1975), which are obtained from the components of the Eshelby's tensor and from the components of the inverse $S_{k l m n}$ of the elasticity tensor by $P_{i j m n}^{0}=E_{i j k l} . S_{k l m n}$. This tensor depends only of the elastic properties of the infinite medium. $Q_{i j m n r s}^{0}, P_{i j k m n r}^{0}, P_{i j k l m n r s}^{0}$ are the components of higher-order Hill-type tensors which are introduced by $a_{i j k}$ and $a_{i j k l}$. As for the classical Hill's tensor, they are built from the inverse of the elasticity tensor and from higher order Eshelby's tensors which can be found in Mura (1987). Those tensors can be derived within the basis composed of $\mathbb{T}_{n}$ for both case of a sixth and eighth order tensor and translated into the basis composed of $\left(\mathbb{K}_{n}, \mathbb{J}_{n}^{p}\right)$ by using the base change relations given in appendix. Note that, once $a_{i j k l}$ is determined by solving the last equation in (5.2), one can compute $c_{i j}$ for obtaining $a_{i j}$ from the first equation in (5.2).

It can be observed that $a_{i j}, a_{i j k}$ and $a_{i j k l}$ are solutions of a linear equation having 
the form:

$$
\boldsymbol{b}=\underbrace{\left[\mathbb{I}-\mathbb{P}^{0} \odot_{n} \delta \mathbb{C}\right]}_{=\mathbb{A}} \odot_{n} \boldsymbol{a}
$$

for $n=2,3,4$. Obviously, a closed-form expression of the strain field within the inclusion requires the inversion of tensor $\mathbb{A}=\mathbb{I}-\mathbb{P}^{0} \odot_{n} \delta \mathbb{C}$ for which it will be convenient to use the formalism introduced in the last sections.

- In the case where $\boldsymbol{a}$ is a second order tensor, $\mathbb{I}, \mathbb{P}^{0}$ and $\delta \mathbb{C}$ are $4^{t h}$-order isotropic tensors having the minor symmetries. The components of $\boldsymbol{b}$ are given by $b_{i j}=$ $a_{i j}-c_{i j}$. Solution of (5.4) is trivial, and can be found in Mura (1987), for instance.

- Consider now the case of a third order tensor $\boldsymbol{a}$. The components of the Hill-type tensor $\mathbb{P}^{0}$ written in the basis $\left(\mathbb{K}_{n}, \mathbb{J}_{n}\right)$ given in section 2 , are:

$$
\begin{aligned}
& P_{1}^{0}=\frac{11-14 \nu_{0}}{35 \mu_{0}\left(1-\nu_{0}\right)}, \quad P_{2}^{0}=\frac{1}{10 \mu_{0}}, \quad P_{3}^{0}=\frac{1-2 \nu_{0}}{10 \mu_{0}\left(1-\nu_{0}\right)} \\
& P_{4}^{0}=-\frac{\nu_{0}}{10 \mu_{0}\left(1-\nu_{0}\right)}, \quad P_{5}^{0}=\frac{1-2 \nu_{0}}{5 \mu_{0}\left(1-\nu_{0}\right)}, \quad P_{6}^{0}=\frac{5-7 \nu_{0}}{10 \mu_{0}\left(1-\nu_{0}\right)}
\end{aligned}
$$

$P_{1}^{0} . . P_{6}^{0}$ are the components of $\mathbb{P}^{0}$ in the basis $\left(\mathbb{K}_{n}, \mathbb{J}_{n}\right)$ as defined in $(2.7)$. The components of $\delta \mathbb{C}$ are $\delta C_{i j p q} \delta_{k r}$. The decomposition in the basis $\left(\mathbb{K}_{n}, \mathbb{d}_{n}\right)$ is given by:

$$
\begin{aligned}
\delta \mathbb{C} & =\delta \lambda \mathbb{T}_{1}+2 \delta \mu \mathbb{T}_{2} \\
& =\delta \lambda\left[3 \mathbb{J}_{1}+\mathbb{J}_{2}\right]+2 \delta \mu\left[\mathbb{K}_{1}+\mathbb{K}_{2}+\mathbb{J}_{1}+\mathbb{J}_{4}\right]
\end{aligned}
$$

The computation of the inverse of $\mathbb{A}=\mathbb{I}-\mathbb{P}^{0} \odot_{n} \delta \mathbb{C}$, denoted $\mathbb{B}$ leads to:

$$
\begin{aligned}
& B_{1}=1+\frac{4\left(\mu_{0}-\mu\right)\left(4 \lambda_{0}+11 \mu_{0}\right)}{16 \lambda_{0} \mu+19 \mu_{0} \lambda_{0}+44 \mu_{0} \mu+26 \mu_{0}^{2}}, \quad B 2=\frac{5 \mu_{0}}{\mu+4 \mu_{0}} \\
& B_{3}=1+\mu D_{1}, \quad B_{4}=D_{2}, \quad B_{5}=2 \mu_{0} D_{1}, \quad B_{6}=\frac{\mu_{0}}{\mu}\left(1+2 D_{2}\right) \\
& D_{1}=\frac{2 \mu_{0}+3 \lambda_{0}-2 \mu-3 \lambda}{3 \lambda \mu+2 \mu_{0} \lambda+2 \mu^{2}+8 \mu_{0} \mu} \\
& D_{2}=\frac{\lambda_{0} \mu-\mu_{0} \lambda}{3 \lambda \mu+2 \mu_{0} \lambda+2 \mu^{2}+8 \mu_{0} \mu}
\end{aligned}
$$


- In the case $n=4$, The Hill-type tensor $\mathbb{P}^{0}$ is decomposed within the basis $\left(\mathbb{K}_{n}, \mathbb{J}_{n}^{1}, \mathbb{J}_{n}^{2}\right)$ given in section 3 . One has:

$$
\begin{aligned}
& P_{1}^{0}=\frac{2}{7 \mu_{0}}, \quad P_{2}^{0}=\frac{2\left(7-9 \nu_{0}\right)}{21 \mu_{0}\left(1-\nu_{0}\right)}, \quad P_{3}^{0}=\frac{1-2 \nu_{0}}{3 \mu_{0}\left(1-\nu_{0}\right)} \\
& P_{4}^{0}=\frac{2\left(1-2 \nu_{0}\right)}{15 \mu_{0}\left(1-\nu_{0}\right)}, \quad P_{5}^{0}=\frac{4\left(1-2 \nu_{0}\right)}{21 \mu_{0}\left(1-\nu_{0}\right)}, \quad P_{6}^{0}=\frac{1-2 \nu_{0}}{3 \mu_{0}\left(1-\nu_{0}\right)} \\
& P_{7}^{0}=\frac{2\left(4-5 \nu_{0}\right)}{15 \mu_{0}\left(1-\nu_{0}\right)}, \quad P_{8}^{0}=\frac{2\left(5-7 \nu_{0}\right)}{21 \mu_{0}\left(1-\nu_{0}\right)}, \quad P_{9}^{0}=\frac{1-2 \nu_{0}}{3 \mu_{0}\left(1-\nu_{0}\right)} \\
& P_{10}^{0}=\frac{5-7 \nu_{0}}{15 \mu_{0}\left(1-\nu_{0}\right)}, \quad P_{11}^{0}=\frac{7-11 \nu_{0}}{21 \mu_{0}\left(1-\nu_{0}\right)}, \quad P_{12}^{0}=\frac{1}{\mu_{0}} \\
& P_{13}^{0}=\frac{1-2 \nu_{0}}{5 \mu_{0}\left(1-\nu_{0}\right)}, \quad P_{14}^{0}=\frac{2\left(1-2 \nu_{0}\right)}{5 \mu_{0}\left(1-\nu_{0}\right)}, \quad P_{15}^{0}=\frac{2\left(1-2 \nu_{0}\right)}{5 \mu_{0}\left(1-\nu_{0}\right)} \\
& P_{16}^{0}=\frac{2\left(1-2 \nu_{0}\right)}{5 \mu_{0}\left(1-\nu_{0}\right)}
\end{aligned}
$$

$P_{1}^{0} . . P_{16}^{0}$ are the components of $\mathbb{P}^{0}$ in the basis $\left(\mathbb{K}_{n}, \mathbb{J}_{n}^{1}, \mathbb{J}_{n}^{2}\right)$ as defined in $(3.12)$. $\delta C_{i j k l}$ is replaced by an equivalent $8^{t h}$-order tensor whose components are given by $\delta C_{i j k l} I_{\text {pqrs }}$ which reads, in the basis $\left(\mathbb{K}_{n}, \mathbb{J}_{n}^{1}, \mathbb{d}_{n}^{2}\right)$ :

$$
\begin{aligned}
\delta \mathbb{C} & =\delta \lambda \mathbb{T}_{2}+2 \delta \mu \mathbb{T}_{13} \\
& =3 \delta \lambda\left[\mathbb{J}_{1}^{2}+2 \mathbb{J}_{2}^{2}\right]+2 \delta \mu\left[\mathbb{K}_{1}+\mathbb{K}_{2}+\mathbb{J}_{1}^{1}+\mathbb{J}_{5}^{1}+\mathbb{J}_{9}^{1}+\mathbb{J}_{10}^{1}+\mathbb{J}_{1}^{2}+\mathbb{J}_{4}^{2}\right]
\end{aligned}
$$

Components of $\mathbb{B}$ are given by:

$$
\begin{aligned}
& B_{1}=1-\frac{4\left(\mu-\mu_{0}\right)}{3 \mu_{0}+4 \mu}, \quad B_{2}=1-\frac{4\left(\mu-\mu_{0}\right)\left(5 \lambda_{0}+14 \mu_{0}\right)}{\left(\lambda_{0} \mu_{0}-14 \mu_{0}^{2}+20 \lambda_{0} \mu+56 \mu \mu_{0}\right)} \\
& B_{3}=1+2 D_{1}\left(3 \mu-38 \mu_{0}\right), \quad B_{4}=4 D_{1}\left(2 \mu-9 \mu_{0}\right) \\
& B_{5}=-8 D_{1}\left(2 \mu+3 \mu_{0}\right), \quad B_{6}=10 D_{1}\left(2 \mu-9 \mu_{0}\right) \\
& B_{7}=1-2 D_{1}\left(32 \mu+21 \lambda_{0}+24 \mu_{0}\right), \quad B_{8}=10 D_{1}\left(4 \mu+3 \lambda_{0}+6 \mu_{0}\right) \\
& B_{9}=-14 D_{1}\left(2 \mu+3 \mu_{0}\right), \quad B_{10}=-7 D_{1}\left(4 \mu+3 \lambda_{0}+6 \mu_{0}\right) \\
& B_{11}=1-D_{1}\left(28 \mu+15 \lambda_{0}+42 \mu_{0}\right), \quad B_{12}=\frac{\mu_{0}}{2 \mu-\mu_{0}} \\
& D_{1}=\frac{2\left(\mu-\mu_{0}\right)}{3\left(-3 \lambda_{0} \mu_{0}+38 \lambda_{0} \mu-42 \mu_{0}^{2}+56 \mu^{2}+56 \mu \mu_{0}\right)} \\
& B_{13}=1+D_{2}, \quad B_{14}=B_{15}=2 D_{2}, \quad B_{16}=1+4 D_{2} \\
& D_{2}=\frac{2}{5} \frac{3\left(\lambda-\lambda_{0}\right)+2\left(\mu-\mu_{0}\right)}{5 \lambda_{0}+2 \mu_{0}-6 \lambda-4 \mu}
\end{aligned}
$$

These results finalize the closed form solution of the higher order "heterogeneity problems". 


\section{Conclusion}

The present study deals about the inversion of an isotropic $2 n^{t h}$-order tensor having particular symmetries (called "minor symmetries" in the paper). To reach this objective, irreducible bases for isotropic $2 n^{t h}$-order tensors has been provided in the present paper. These bases extend the $(\mathbb{J}, \mathbb{K})$ basis used for isotropic $4^{\text {th }}$-order tensors. The particular case of $6^{t h}$-order and $8^{t h}$-order tensors has been examined in this paper and higher-order cases has been addressed in section 4 . The methodology used consists in decomposing $3^{r d}$-order and $4^{\text {th }}$-order tensors into their deviatoric and spherical parts as commonly used in the case of a tensor of order 2 . The particularity with tensors of order $n \geq 4$ lies in the definition of spherical parts of order $1,2,3 \ldots$ while for tensors of order $n \leq 3$ only one definition of the spherical part is used (for instance, in the case of a $2^{\text {nd }}$-order tensor the first spherical part corresponds to the classical definition). This decomposition of a $n^{\text {th }}$ tensor appears to be useful for obtaining the irreducible bases " $\left(\mathbb{K}_{n}, \mathbb{J}_{n}^{p}\right)$ " for isotropic $2 n^{t h}$-order tensors.

It it shown that the bases " $\left(\mathbb{K}_{n}, \mathbb{J}_{n}^{p}\right)$ " are useful for effecting the tensorial operations and particularly for the inversion of a $2 n^{t h}$-order tensor. In order to show the relevance of this formalism, we derive the closed-form expression of the strain field within a spherical inclusion subjected to a polynomial remote strain field. This result is an extension of the well known use of the Eshelby's tensor for obtaining the solution of the heterogeneity problem which is the base of numerous homogenization problems. It suggests that the results of higher order heterogeneity problems could be used for obtaining the effective properties in the context of gradient elasticity. This will be developped in a forthcoming paper.

\section{Appendix A. The SFH decomposition}

The SFH decomposition of a third order tensor $\boldsymbol{a}$ (symmetric according to its two first indices) has been introduce by Smyshlyaev \& Fleck (1996) and formalized by Fleck \& Hutchinson (1997). It reads:

$$
\boldsymbol{a}=\boldsymbol{a}^{(1)}+\boldsymbol{a}^{(2)}+\boldsymbol{a}^{(3)}
$$

with:

$$
\begin{aligned}
& a_{i j k}^{(1)}=a_{i j k}^{s}-\frac{1}{5}\left(\delta_{i j} a_{k p p}^{s}+\delta_{i k} a_{j p p}^{s}+\delta_{j k} a_{i p p}^{s}\right) \\
& a_{i j k}^{(2)}=\frac{1}{3}\left(\varepsilon_{k i p} \kappa_{p j}^{s}+\varepsilon_{k j p} \kappa_{p i}^{s}\right) \\
& a_{i j k}^{(3)}=\frac{1}{3}\left(\varepsilon_{k i p} \kappa_{p j}^{a}+\varepsilon_{k j p} \kappa_{p i}^{a}\right)-\frac{1}{5}\left(\delta_{i j} a_{k p p}^{s}+\delta_{i k} a_{j p p}^{s}+\delta_{j k} a_{i p p}^{s}\right) \\
& a_{i j k}^{s}=\frac{1}{3}\left(a_{i j k}+a_{i k j}+a_{j k i}\right), \quad \kappa_{p j}^{s}=\frac{1}{2}\left(\kappa_{i j}+\kappa_{j i}\right), \quad \kappa_{p j}^{a}=\frac{1}{2}\left(\kappa_{i j}-\kappa_{j i}\right) \\
& \kappa_{i j}=\varepsilon_{i p q} a_{j q p}
\end{aligned}
$$

where $\varepsilon_{i j k}$ is the permutation symbol. 


\section{Appendix B. Base change relations}

Let us denote by $a_{n}$ for $n=1 . .6$ the components of a sixth order tensor $\mathbb{A}$ in the basis $\mathbb{T}_{n}$ for $n=1 . .6$. Let the $b_{n}$ for $n=1 . .6$ be the components of $\mathbb{A}$ in the basis $\left(\mathbb{K}_{n}, \mathbb{J}_{n}\right)$ as defined in (2.7). The relations giving the $b_{n}$ as functions of the $a_{n}$ are:

$$
\begin{aligned}
& b_{1}=a_{2}+a_{5} \\
& b_{2}=a_{2}-a_{5} / 2 \\
& b_{3}=3 a_{1}+a_{2}+a_{4} \\
& b_{4}=a_{1}+2 a_{4}+a_{5} / 2 \\
& b_{5}=3 a_{3}+a_{5}+a_{6} \\
& b_{6}=a_{2}+a_{3}+a_{5} / 2+2 a_{6}
\end{aligned}
$$

Let us denote by $a_{n}$ for $n=1 . .16$ the components of an eighth order tensor $\mathbb{A}$ in the basis $\mathbb{T}_{n}$ for $n=1 . .16$ and let the $b_{n}$ for $n=1 . .16$ its components in the basis $\left(\mathbb{K}_{n}, \mathbb{J}_{n}^{1}, \mathbb{J}_{n}^{2}\right)$ as defined in $(3.12)$. The relations giving the $b_{n}$ as function of the $a_{n}$ are:

$$
\begin{aligned}
& b_{1}=a_{13}-a_{14} \\
& b_{2}=a_{13}+a_{14}+a_{15} \\
& b_{3}=3 a_{3}+a_{8}+a_{10}+a_{13}+\left(a_{15}+a_{16}\right) / 3 \\
& b_{4}=3 a_{4}+a_{8}+a_{11}+a_{14}+\left(a_{15}+a_{16}\right) / 3 \\
& b_{5}=a_{8}+\left(a_{15}+a_{16}\right) / 3 \\
& b_{6}=3 a_{5}+a_{9}+a_{10}+a_{14}+\left(a_{15}+a_{16}\right) / 3 \\
& b_{7}=3 a_{6}+a_{9}+a_{11}+a_{13}+\left(a_{15}+a_{16}\right) / 3 \\
& b_{8}=a_{9}+\left(a_{15}+a_{16}\right) / 3 \\
& b_{9}=7\left(3 a_{10}+a_{15}+a_{16}\right) / 12 \\
& b_{10}=7\left(3 a_{11}+a_{15}+a_{16}\right) / 12 \\
& b_{11}=a_{13}+a_{14}-a_{15} / 6+7 a_{16} / 12 \\
& b_{12}=a_{13}-a_{14}-5 a_{16} / 4 \\
& b_{13}=9 a_{1}+3 a_{2}+3 a_{3}+3 a_{4}+3 a_{5}+3 a_{6}+a_{8}+a_{9}+a_{13}+a_{14} \\
& b_{14}=3 a_{1}+6 a_{2}+a_{3}+a_{4}+a_{5}+a_{6}+2 a_{8}+2 a_{9}+a_{15} / 2 \\
& b_{15}=9 a_{7}+3 a_{10}+3 a_{11}+3 a_{12}+a_{15}+a_{16} \\
& b_{16}=3 a_{7}+a_{10}+a_{11}+6 a_{12}+a_{13}+a_{14}+a_{15} / 2+2 a_{16}
\end{aligned}
$$

\section{References}

Asaro, R. J. \& Barnett, D. M. 1975 The non-uniform transformation strain problem for an anisotropic ellipsoidal inclusion. J. Mech. Phys. Solids 23 1, 77-83.

Dell'Isola, F., Sciarra, G., Vidoli, S. 2009 Generalized Hooke's law for isotropic second gradient materials. Proc. R. Soc. A 465, 2177-2196. 
Eshelby, J. D. 1957 The determination of the elastic field of an ellipsoidal inclusion, and related problem. Proc. R. Soc. A 241, 376-396.

Fleck, N. A., \& Hutchinson, J. W. 1997 Strain gradient plasticity. Adv. Appl. Mech. 33, 295-361.

Gurtin, M. E. \& Anand, L. 2005 A theory of strain-gradient plasticity for isotropic, plastically irrotational materials. Part I : Small deformations. J. Mech. Phys. Solids 53, 1624-1649.

Hill, R. 1975 Continuum micromechanics of elastoplastic polycrystals. J. Mech. Phys. Solids 13, 89-101.

Kearsley, E. A. \& Fong, J. T. 1975 Linearly independent sets of isotropic Cartesian tensors of ranks up to eight. Journal of Research of the National Bureau of Standards Part B: Mathematical Sciences. 79B, 49-58.

Lubarda, V. A. \& Krajcinovic, D. 1993 Damage tensors and the crack density distribution. Int. J. Solids Struct. 30 20, 2859-2877.

Mindlin, R. D. 1964 Micro-structure in linear elasticity. Arch. Rat. Mech. Anal. 16, 51-78.

Mindlin, R. D. \& Eshel, N. N. 1968 On first strain-gradient theory in linear elasticity. Int. J. Solids Struct. 4, 109-124.

T. Mura. 1987 Micromechanics of Defects in Solids. Dordrecht: Martinus Nijhoff.

Smyshlyaev, V. P. \& Fleck, N. A. 1996 The role of strain gradients in the grain size effect for polycrystals. J. Mech. Phys. Solids 44, 465-496.

Suiker, A. S. J. \& Chang, C. S. 2000 Application of higher-order theory for formulating enhanced continuum models. Acta Mech. 142, 223-234.

Toupin, R. 1962 Elastic materials with couple-stresses. Arch. Rat. Mech. Anal. 11 1, 385414. 